\title{
Infeção por Chlamydia pneumoniae e Hepatite Aguda
}

\author{
Chlamydia pneumoniae infection and Acute Hepatitis
}

\author{
Ana Raquel Ramos, Miguel Moreira, Ana Mondragão, Margarida Correia \\ Serviço de Medicina Interna, Centro Hospital Vila Nova de Gaia/Espinho, Portugal
}

\section{RESUMO}

A Chlamydia pneumoniae é uma espécie associada sobretudo a infeções da via aérea respiratória, e pouco descrita e muito raramente associada a Hepatite Aguda. Apresenta-se um caso de uma mulher de 86 que recorreu ao Serviço de Urgência por quadro de epigastralgias, associadas a náuseas e vómitos pós prandiais. Ao exame objetivo: subfebril e 0 abdómen globoso, com timpanismo difuso e doloroso à palpação profunda do epigastro e hipocôndrio direito. Do estudo complementar realizado apresentava parâmetros inflamatórios aumentados, uma citocolestase hepática, sem insuficiência hepática, com ecografia abdominal apenas com ectasia da via biliar principal; e excluiu-se causa tóxica, isquémica e imunológica, marcadores víricos negativos; serologias negativas, excepto a serologia da Chlamydia pneumoniae, IgM e IgG positivos. Quadro compatível com uma hepatite colestática aguda sem evidência de infeção respiratória prévia ou concomitante. Os autores apresentam este caso clínico dados a raridade desta correlação, mesmo após exclusão de todas as outras causas.

Palavras chave: hepatite aguda, chlamydia pneumoniae, infeção

\section{INTRODUÇÃO}

A Chlamydia pneumoniae é uma bactéria gram negativa intracelular, a sua transmissão é através da inalação de pequenas partículas de indivíduos infetados. É um agente que causa infeções respiratórias das vias aéreas superiores, geralmente menos grave, e cerca de 5-10\% das pneumonias adquiridas na comunidade. ${ }^{1,2}$ Mais raramente pode provocar derrame pleural; e mais graves, síndrome respiratória aguda, choque sético e insuficiência multiorgânica; e extrarespiratórios, tais como 0 eritema nodoso, meningite, hepatite, vasculite, síndrome de Guillain-Barré, lesão renal aguda, pericardite, miocardite ou síndrome febril de causa não conhecida. Além disso, descreve-se uma possível relação causal entre este microorganismo a doença aterotrombótica e cirrose biliar primária. A hepatite aguda clínica causada por este microorganismo é excecional.,34

\section{CASO CLÍNICO}

Mulher de 86 anos, caucasiana. Com antecedentes de diabetes mellitus tipo 2, dislipidemia e hipertensão arterial, estando medicada há vários anos com glimepirida, furosemida, amlodipina, ácido acetilsalicílico, nitrato, pantoprazol e dislipina. Recorreu ao Serviço de Urgência por quadro de epigastralgias, associadas a náuseas e vómitos pós prandiais. Negava alterações do trânsito intestinal, genitourinárias ou febre. Negava ingestão de chás ou novos medicamentos. Ao exame objetivo: tensão arterial 160/71 mmHg, frequência cardíaca 82bpm, saturação periférica de 02 96\% com Fi02 0,21, subfebril $\left(37.3^{\circ} \mathrm{C}\right)$ e 0 abdómen globoso, com timpanismo difuso e doloroso à palpação profunda do epigastro e hipocôndrio direito. Analiticamente presença de leucocitose $\left(20,57 \times 10^{3} \mathrm{U} / \mathrm{L}\right.$; neutrofilia

\section{ABSTRACT}

Chlamydia pneumoniae is a species mainly associated with infections of the respiratory airway, and little described and very rarely associated with Acute Hepatitis. We present a case of a woman of 86 who presented to the emergency department by epigastralgias, associated with postprandial nausea and vomiting. At the objective examination: subfebrile and the with a diffuse and painful tympanism abdomen to the deep palpation of the epigastrium and right hypochondrium. From the complementary study, he presented increased inflammatory parameters, a hepatic cytocholasease, without hepatic insufficiency, with abdominal ultrasound only with ectasia of the main biliary tract; excluded toxic, ischemic and immunological causes, negative viral markers; Negative serology except Chlamydia pneumoniae serology, $\lg \mathrm{M}$ and $\lg G$ positive. Frame is compatible with acute cholestatic hepatitis with no evidence of prior or concomitant respiratory infection. The authors present this clinical case due to the rarity of this correlation, even after exclusion of all other causes.

Keywords: acute hepatitis, chlamydia pneumoniae, infection

Tabela 1. Estudo etiológico da hepatite aguda

\begin{tabular}{|c|c|}
\hline \multicolumn{2}{|c|}{ Do estudo etiológico } \\
\hline Parâmetros inflamatórios aumentados & Acs anti-Ag hepáticos negativos \\
\hline VS aumentada $68 \mathrm{~mm}$ & $\begin{array}{l}\text { Ac anti-cardiolipina e anti-beta-2 } \\
\text { glicoproteina I negativos }\end{array}$ \\
\hline AST $2122 \mathrm{U} / \mathrm{L}, \mathrm{ALT} 1301 \mathrm{U} / \mathrm{L}$ & Virus Ebstein-Barr negativo \\
\hline FA 134U/L, YGT 415U/L & Citomegalovirus negativo \\
\hline Desidrogenase láctica $1787 \mathrm{U} / \mathrm{L}$ & Toxoplasmose negativo \\
\hline BT $1,14 \mathrm{mg} / \mathrm{dL}, \mathrm{BD} 0,82 \mathrm{mg} / \mathrm{dL}$ & TPHA negativo com VDRL não reactivo \\
\hline Ferritina $2339 \mathrm{ng} / \mathrm{mL}$ & Virus herpes simplex negativo \\
\hline aPTT 27seg, INR 1,14 & $\begin{array}{c}\text { Varicela-zoster, } \\
\text { Sarampoe Rubéola negativos }\end{array}$ \\
\hline electroforese proteínas normal & Virus coxsackie negativos \\
\hline cobre $75 \mathrm{ug} / \mathrm{dL}$, ceruplasmina $20 \mathrm{mg} / \mathrm{dL}$ & Leptospira negativos \\
\hline alfa-fetoproteina negativa $(0,76)$ & $\begin{array}{l}\text { Reacção Weil-Felix, Widal, Wright } \\
\text { negativas }\end{array}$ \\
\hline $\begin{array}{l}\text { hemoculturas aerobiose, anerobiose e fungos } \\
\text { negativas }\end{array}$ & Mycoplasma pneumonia negativo \\
\hline Hepatite A, B, C, D $\Theta E$ negativos & Borrelia negativa \\
\hline VIH 1 e 2 negativos & Coxiella burnetti negativa \\
\hline ANA $<1 / 80$, ant-dsDNA $1,7 \mathrm{Ul} / \mathrm{mL}$ & Chlamydia trachomatis negativa \\
\hline $\begin{array}{c}\text { ANCA }<1 / 40 \text {, anti-mitocóndria } 1 / 40 \text {, anti- } \\
\text { músculo liso }<1 / 40\end{array}$ & $\begin{array}{c}\text { Chlamydia pneumonia positiva (IgM } \\
1 / 160 \text { e IgG } 1 / 800\end{array}$ \\
\hline
\end{tabular}




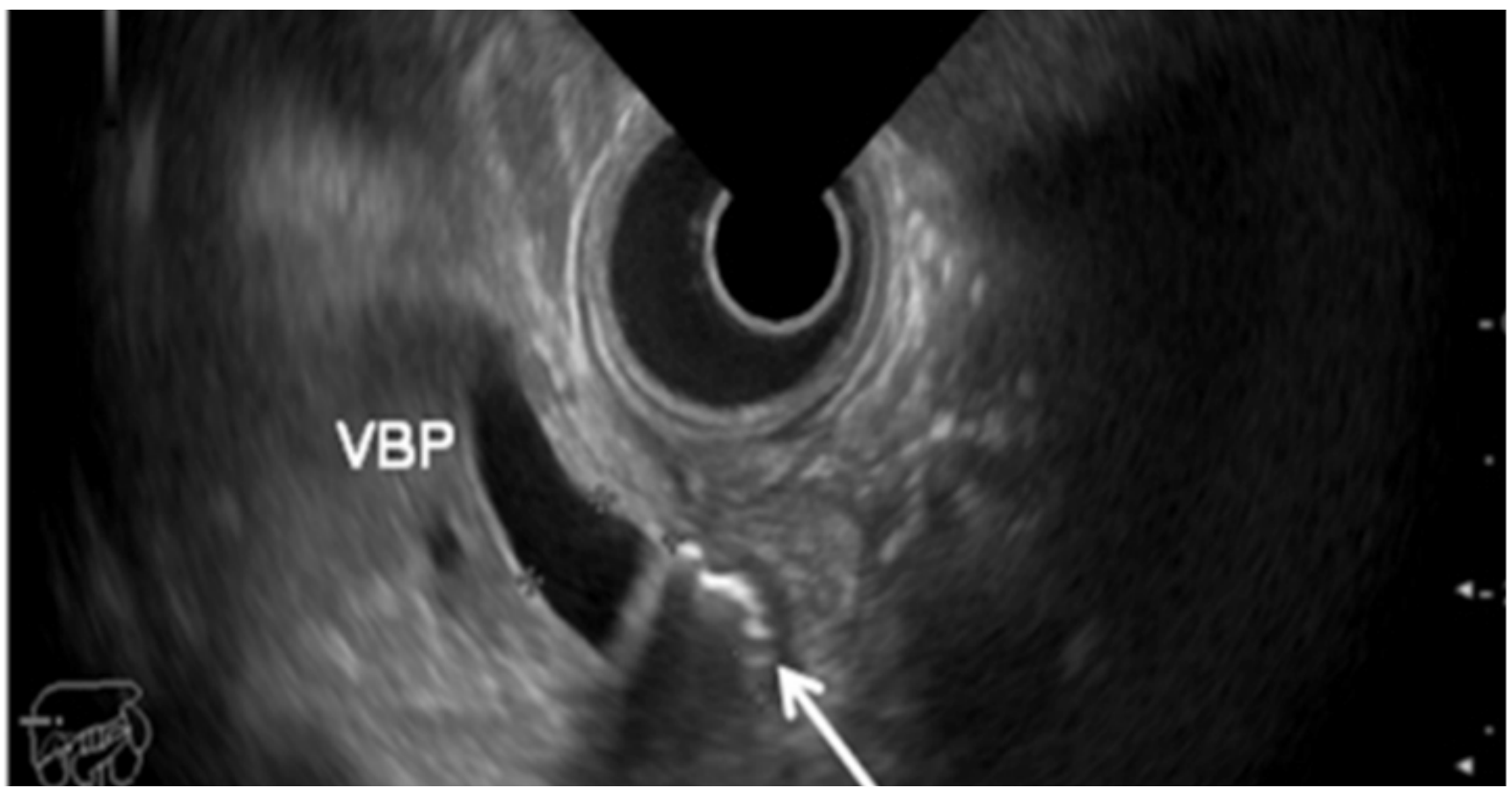

(91,8\%); Proteína C Reativa (PCR) 20,29mg/dL; aPTT 27seg, taxa protrombina $80 \%$ e INR 1,14, aspartato aminotransferase (AST) de 2122 U/L; alanina aminotransferase (ALT) de 1301 U/L; desidrogenase láctica (DHL) de $1787 \mathrm{U} / \mathrm{L}$; bilirrubina total (BT) 1,14 mg/dL com bilirrubina direta (BD) de 0,82mg/dL; fosfatase alcalina (FA) de $134 \mathrm{U} / \mathrm{L}$; gamaglutaminatransferase (YGT) de $415 \mathrm{U} / \mathrm{L}$, ferritina de $2339 \mathrm{ng} / \mathrm{mL}$ e lactatos 0,7. Do estudo efetuado: eletroforese proteínas normal, velocidade de sedimentação (VS) $68 \mathrm{mmHr}$, cobre $75 \mathrm{ug} / \mathrm{dL}$, ceruplasmina $20 \mathrm{mg} / \mathrm{dL}$, alfa-fetoproteína negativa $(0,76)$, hemoculturas aerobiose, anerobiose e fungos negativas. Marcadores víricos: imunização para hepatite A (AcHAV IgG positivo e lgM negativo), hepatite $B$ negativo (AgHBS negativo, $\mathrm{AcHBC}$ negativo, AgHBs negativo), hepatite $C$ negativo (ACHCV negativo), hepatite $D$ e E negativos, e VIH negativo ( $A c+A g V I H 1$ e 2 negativos). Serologias: vírus Epstein-Barrnegativo (VCA IgM negativo, IgG positivo, EBNA IgG positivo e EA IgG positivo), imunização para citomegalovírus (IgM negativo e lgG positivo) e toxoplasmose (IgM negativo, IgG positivo), TPHA negativo com VDRL não reativo, vírus herpes simplex, vírus varicela-zoster, sarampo, rubéola e vírus coxsackie negativos, reação Weil-Felix, Widal, Wright negativas, leptospira negativa (IgM negativo e lgG $<10 \mathrm{U} /$ $\mathrm{mL}$ ), mycoplasma pneumoniae negativo (lgM e lg G negativos), borrelia negativa (IgM e IgG negativos), brucela negativa (IgM e IgG negativos), coxiella burnetti negativa (IgM e lgG negativos), chlamydia trachomatis negativa (IgM e lgG negativos) e chlamydia pneumoniae positiva (IgM 1/160 e lgG 1/800). Estudo auto-imune: complemento C3 (99,5mg/dL) e C4 $(26,3 \mathrm{mg} /$ $\mathrm{dL}$ ) normalizados, imunoglobulinas A, M, G normalizadas, fator reumatóide negativo $(<10,2 \mathrm{Ul} / \mathrm{mL})$, anticorpos anti-nucleares negativos (ANA $<1 / 80$, anti-dsDNA 1,7Ul/mL), anti-citoplasma neutrófilo negativo (ANCA $<1 / 40$, anti-mitocôndria 1/40, anti-músculo liso <1/40), anticorpos anti-Ag hepáticos, anticorpo anti-cardiolipina e anti-beta-2 glicoproteína I negativos. (Tabela 1) A ecografia abdominal mostrava ectasia da via biliar principal, com moderada repercussão intrahepática, não se registando causas obstrutivas. Acentuada distensão da vesícula biliar e presença de discretas imagens hipodensas justacapsulares no segmento VIII, que poderão apenas traduzir heterogeneidades do parênquima, não parecendo configurar verdadeiros nódulos (Figura 1). Concluiu-se, assim, tratar de uma Hepatite Aguda num doente com síndrome de resposta inflamatória sistémica e subfebril, mas sem foco infecioso conhecido, decidiu-se medicar empiricamente com piperacilina/tazobactam $4,5 \mathrm{~g}$ 6/6h endovenoso, que suspendeu no $3^{\circ}$ dia internamento após resultado serológico, e doxiciclina 100mg oral 1x/dia, que cumpriu durante 7 dias. Assim, do estudo etiológico, excluiu-se causa tóxica, isquémica e imunológica; realçando a positividade da serologia da chlamydia pneumoniae, lgM e IgG positivos. A telerradiografia do tórax sem alterações. Teve uma evolução favorável, assintomática, com apirexia sustentada, normalização dos parâmetros inflamatórios; AST 21 U/L, ALT 28 U/L, FA 77 U/L, YGT 129 U/L, BT 0,37mg/dL; VS 28, ferritina 318,5ng/mL (Tabela 2) e ecografia abdominal apenas com discreta distensão da vesícula biliar.

\section{DISCUSSÃO}

A Chlamydia pneumoniae é uma espécie descrita que, conforme o próprio nome indica, está associada sobretudo a infeções da via aérea respiratória. Inicialmente, acreditava-se que as estirpes da Chlamydia pneumoniae eram variantes da Chlamydia psittaci, mas estudos subsequentes demonstraram 
Tabela 2. Estudo analítico na alta

\begin{tabular}{|c|}
\hline Do estudo analitico na alta \\
\hline Parâmetros inflamatórios normalizados \\
\hline AST $21 \mathrm{U} / \mathrm{L}$ \\
\hline ALT $28 \mathrm{U} / \mathrm{L}$ \\
\hline BT $0,37 \mathrm{mg} / \mathrm{dL}$ \\
\hline FA $77 \mathrm{U} / \mathrm{L}$ \\
\hline YGT $129 \mathrm{U} / \mathrm{L}$ \\
\hline Ferritina $318,5 \mathrm{ng} / \mathrm{mL}$ \\
\hline
\end{tabular}

que a Chlamydia pneumoniae constitui uma espécie distinta tomando como base a existência de pequenas diferenças, envolvendo uma pequena sequência de subunidade de RNA ribossómico, a morfologia e as propriedades antigénicas. ${ }^{2} \mathrm{~A}$ Chlamydia pneumoniae originada de amostras clínicas, cresce de maneira mais efetiva em células HL e em células Hep-2. Devido à dificuldade para isolar o organismo, a maioria dos estudos epidemiológicos dependem da realização de ensaios serológicos de microimunofluorescência. Tais estudos indicam que a infeção causada pela Chlamydia pneumoniae é extremamente prevalente, quando nos referimos às infeções respiratórias, com cerca de 40 a $75 \%$ dos adultos, apresentando serologias positivas em praticamente todos os países a nível mundial. Mas, pouco se sabe ainda sobre a patogénese molecular da infeção por Chlamydia pneumoniae. Entretanto, acredita-se que o organismo no início promova infeção do epitélio da via aérea respiratória superior. Mas em muitos indivíduos, a infeção é assintomática e persiste nestes locais. Estudos recentes demonstraram que após a infeção das células epiteliais da via aérea respiratória e das células inflamatórias, é provável que a Chlamydia pneumoniae se dissemine pelo organismo através dos macrófagos presentes na circulação sanguínea. Existem evidências nítidas de que a replicação do organismo ocorre no endotélio vascular e nas membranas sinoviais. Assim, a proteína da membrana externa da Chlamydia pneumoniae pode induzir respostas imunes do hospedeiro que apresentam reação cruzada com proteínas humanas e, consequentemente, produzem lesão inflamatória autoimune aos tecidos. ${ }^{5}$ Além das doenças respiratórias, a Chlamydia pneumoniae foi recentemente associada, em estudos serológicos, a uma ampla gama de condições distintas, como miocardite, pericardite, meningite assética, febre de causa não conhecida, eritema nodoso, sarcoidose, asma, hepatite, esclerose múltipla e doença de Alzheimer. No entanto, a validade destas associações continua incerta. ${ }^{4}$ Outros estudos identificam chlamydia pneumoniae no líquido cefalorraquidiano por reação em cadeia da polimerase em doentes com esclerose múltipla definida ${ }^{6}$; e outro estudo, este em animais experimentais (ratos) mostra que o péptido derivado da chlamydia pneumoniae é capaz de induzir doença do sistema nervoso central auto-imune, logo assume que a chlamydia pneumoniae desempenha um papel na patogénese da esclerose múltipla, mas sem estudos ainda validados em humanos. ${ }^{7}$ Num estudo mais recente de 2005, tentam mostrar a associação da infeção por chlamydia pneumoniae e a esclerose múltipla em humanos. 0 estudo revelou a presença de DNA chlamydia pneumoniae no líquido cefalorraquidiano em 0\% ->180\% de doentes com esclerose múltipla e entre $0 \%$ - 20\% de amostras de líquido cefalorraquidiano de doentes com outras doenças neurológicas. Estas discrepâncias podem indicar diferenças metodológicas, erro de amostragem ou outros problemas desconhecidos. Vários estudos multicêntricos demonstraram diferenças significativas em metodologias e resultados, incluindo significativa variabilidade inter e intra laboratório nos testes de PCR. Assim, mais estudos são necessários antes que possamos inferir cientificamente uma relação biológica entre a infeção por chlamydia pneumoniae e a esclerose múltipla. ${ }^{8}$ Com este estudo podemos considerar que a chlamydia pneumonia pode ter um papel na patogénese da esclerose múltipla mas ainda é incerto.

As infeções continuam a ser a causa principal de febre de causa não conhecida (30\%-40\%), localizada ou generalizada. Dentro de infeções bacterianas sistémicas, a infeção por chlamydia pneumoniae pertence ao fim da lista de causas de febre de causa não conhecida. Mas, estudos têm vindo mostrar que a relação entre esta entidade e este microorganismo. A ausência de casos de infeção por chlamydia pneumoniae não permite a conclusão de que eles não existam no nosso meio, mas provavelmente permanecem sem diagnóstico. Estas formas clínicas são normalmente incluídas nos diagnósticos autolimitados de febre sem causa conhecida. Assim, a chlamydia pneumoniae deve ser considerada no diagnóstico diferencial da febre sem causa conhecida, na tentativa de minimizar gastos e técnicas menos invasivas para os doentes. ${ }^{9}$ No caso em questão, estamos perante uma doente com uma hepatite. Sabemos da literatura que a etiologia da hepatite aguda é diversificada, a maioria são devido a infeções por vírus e por fármacos hepatotóxicos, seja mecanismos dependentes da dose ou idiossincrática. Da revisão realizada, verificou-se a não exposição a novos fármacos, nem ingestão de chás ou alimentos de novo na dieta; sem perfil hipotensivo, sem hipoxemia com lactatos dentro dos valores normais, sem alterações do ritmo cardíaco, nem perdas hemáticas visíveis que possam sugerir hipovolémia/hipoperfusão de órgão, excluindo deste modo as causas tóxicas e isquémicas. Entre as causas infeciosas para além da hepatite $A, B, C, D$ e E, os outros vírus não especificamente hepatotrópicos (Epstein-Bar, citomegalovírus, herpes simplex, vírus varicela-zoster, sarampo, rubéola e vírus Coxsackie) foram excluídos; assim como, bacteriémia e fungémia que também, geralmente, apresentam atingimento hepático. Outros agentes infeciosos, tais como coxiella burnetii, brucela, leptospira, micobactérias pode, também causar hepatite, no contexto de uma doença sistémica. Estes últimos também foram descartados.

Existem estudos que indicam que a Chlamydia pneumoniae pode persistir em hepatócitos e, mais recentemente, relataram a presença de antígenos de Chlamydia pneumoniae e RNA ribossómico nos hepatócitos de doentes com cirrose biliar primária em fase terminal, sugerindo um papel potencial da Chlamydia pneumonia na etiologia e patogénese da doença. 
Assim, foram produzidas evidências quanto à possível localização e/ou replicação da Chlamydia pneumonia em células hepáticas parenquimatosas e não parenquimatosas, principalmente em células de Kupffer, onde clamídia poderia atuar como um trigger celular imunológico. A capacidade da Chlamydia pneumoniae sobreviver e replicar nas células de Kupffer, demonstra a capacidade desta bactéria para escapar, sob certas condições, os mecanismos de defesa da imunidade natural. Esta caraterística pode favorecer a produção de substratos altamente imunogénicos e/ou fornecer um microambiente inflamatório para aumentar a auto reatividade de linfócitos T patogénicos, ou pode ativar o repertório celular pré-existente. ${ }^{10}$ Relativamente as causas imunes, excluída a hepatite auto-imune, pois a doente apresenta títulos de ANA $<1 / 80$, anti-músculo liso $<1 / 40$ e Ac anti LKM-1 negativo; e mais raro mas não menos importantes as entidade cirrose biliar primária e colangite esclerosante primária, a primeira não se enquadra na clínica da doente, apenas com citocolestase, e com ANA <1/80, IgM normalizado e anti-mitocôndria 1/40; a segunda entidade normalmente está associada a doença inflamatória intestinal, que a doente não refere e tem ANA $<1 / 80$ e ANCA $<1 / 40$ negativos..$^{11}$ Assim, apraz dizer-me que etiologia imune não justifica a hepatite aguda do caso. Deste modo, o caso apresentado mostra uma hepatite colestática aguda sem evidência de infeção respiratória prévia ou concomitante, com boa evolução espontânea e para 0 qual não foi possível encontrar um diagnóstico alternativo. Logo, a positividade para chlamydia pneumoniae (IgM 1/160 e IgG 1/800) faz plausível 0 diagnóstico da infeção aguda por este organismo. Portanto, acreditamos que a Chlamydia pneumoniae deve ser considerada no diagnóstico diferencial de hepatite aguda, especialmente quando os outros agentes mais comuns são excluídos.

\section{REFERÊNCIAS}

1. Prieto JM, Hidalgo S., Bouza JM, Rebollo ML, Hepatitis aguda por Chlamydophila pneumoniae, Rev Clin Esp, 2011; 211:607-8.

2. Grayston JT, Aldous MB, Easton A, Wang SP, Kuo CC, Campbell LA, Altman J, Evidence that Chlamydia pneumoniae causes pneumonia and bronchitis, J Infect Dis 1993; 168:1231-1235.

3. Kalambokis G, Ekonomou M, Kitsanou A, Kostoula C, Bobojianni E, Extrarespiratory Chlamydia pneumoniae infection associated with immune disorder, hepatitis and renal disease, Scand J Infect Dis, 2003; 35:424-6.

4. Sundelöf $H$, Gnarpe J, Gnarpe B, An unusual manifestation of Chlamydia pneumoniae infection: meningitis, hepatitis, iritis and atypical erythema nodosum, Scand J Infect Dis, 1993; 25:259-61.

5. Gaydos JT, Summergill NN, Sahney JA, Ramírez TC, Replication of Chlamydia pneumoniae in vitro in human macrophages, endotelial cells and aortic artery smooth muscle cell, Infect Immun, 1996; 64:1414-20.

6. Layh-Schmitt G, Bendl C, Hildt U, Dong-Si T, Jüttler E, Schnitzler P, Evidence for infection with Chlamydia pneumoniae in a subgroup of patients with multiple sclerosis, Ann Neurol 2000; 47:652-655.

7. Lenz DC, Lu L, Conant SB, Wolf NA, Gerard HC, Whittum-Hudson JA, Hudson AP, Swanborg RH, A Chlamydia pneumoniae-specific peptide induces experimental autoimmune encephalomyelitis in rats, J Immunol 2001; 167:1803-1808.

8. Hammerschlag MR, Apfalter P, Boman J, Tondella ML, Gaydos C, The Role of Chlamydia pneumoniae in Multiple Sclerosis: Real or Fictitious?, J Infect Dis 2005; 192:1305-1307.

9. Aranda A, Síndrome febril prolongado e infección por Chlamydia pneumoniae, Rev Clin Esp, 2003; 203(8):382-3.

10. Liu HY, Deng AM, Zhang J, Zhou Y, Yao DK, Tu XQ, Fan LY, Zhong RQ, Correlation of Chlamydia pneumoniae infection with primary biliary cirrhosis. World J Gastroenterol $2005 ; 11: 4108-4110$.

11. Ljungh A, Nilsson HO, Wadstrom $T$, Stenram U, Willen R, Microbes as trigger for primary biliary cirrhosis and primary sclerosing cholangitis, Dig Liver Dis 2005: 37:803-804. 